\title{
Hyperspectral terahertz imaging for human bone biometrics
}

\author{
Suzanna Freer ${ }^{\mathrm{a}}$, Cong Sui ${ }^{\mathrm{b}}$, Pavel Penchev ${ }^{\mathrm{c}}$, Stefan Dimov ${ }^{\mathrm{c}}$, Andrei Gorodetskya,d, Stephen M. \\ Hanham $^{\mathrm{e}}$, Liam M. Grover ${ }^{\mathrm{b}}$, and Miguel Navarro-Cía ${ }^{\mathrm{a}, \mathrm{e}}$ \\ aSchool of Physics and Astronomy, University of Birmingham, UK \\ ${ }^{b}$ Department of Chemical Engineering, University of Birmingham, UK \\ ${ }^{\mathrm{c}}$ Department of Mechanical Engineering, University of Birmingham, UK \\ ${ }^{\mathrm{d}}$ ITMO University, Russia \\ ${ }^{\mathrm{e}}$ Department of Electronic, Electrical and Systems Engineering, University of Birmingham, UK
}

\begin{abstract}
The realisation of hyperspectral terahertz imaging is a significant step towards understanding of the life sciences on all scales. A key to this understanding is the retrieval of dielectric properties from such images, a task which is plagued by experimental limitations, challenging the terahertz community for more than two decades. In this contribution, we propose a new combined retrieval methodology to overcome misalignments and Fabry-Pérot effects on the extraction of the dielectric properties of human bone samples through the combination of the Kramers-Kronig relations and Fabry-Pérot reflection modelling.

Results extracted from $\sim 100 \mu \mathrm{m}$ human bone slices composed largely of collagen are consistent with those measured for pristine collagen samples. This represents another stepping-stone towards the adoption of terahertz imaging into pre- and clinical practice.
\end{abstract}

Keywords: Terahertz, time domain spectroscopy, dielectric imaging, human bone, Kramers-Kronig relations, Fabry-Pérot effects, dielectric property extraction

\section{INTRODUCTION}

The emerging discipline of terahertz ( $f=0.1$ to $10 \mathrm{THz}$ ) imaging has found niche applications in security, surveillance and non-destructive testing, ${ }^{1,2}$ but is still identifying opportunities to exhibit its benefits in terms of spatial resolution and non-invasive measurement of hydration level and conductivity to the life sciences community. ${ }^{3,4}$ Particular attention has been drawn towards tissue disease diagnosis, utilising the high water content of the human body together with the highly absorbing properties of water at terahertz frequencies. This extends to diseases in human bones, such as metastatic defects and osteoporosis, with further application to prosthesis design..$^{5,6}$

A large body of fundamental research has been carried out in the last two decades demonstrating the ability of terahertz time-domain spectroscopic imaging to provide true biometric information. Using such work as a springboard, some translational research is now at an initial stage and practical challenges are surfacing. ${ }^{7}$ Given the high absorption of water, researchers have been forced to turn from transmission spectroscopy to reflection configurations. Common practical challenges that affect reflection retrieval methods used to extract ancillary biometric information in the form of complex permittivity include misalignments ${ }^{8,9}$ and Fabry-Perot effects in thin samples. ${ }^{10,11}$

In this contribution, we report biometric information of $\sim 100 \mu \mathrm{m}$ human bone slices composed largely of collagen, for prospective disease identification (see Fig. 1). The hybrid extraction algorithm involves application of the Kramers-Kronig relations to correct experimental misalignment, in addition to an optimisation method specifically designed to cancel Fabry-Perot interference effects of optically thin dispersive samples. ${ }^{12}$

Further author information: (Send correspondence to M. Navarro-Cía)

M. Navarro-Cía: E-mail: m.navarro-cia@bham.ac.uk, Telephone: +44 (0) 1214144664 

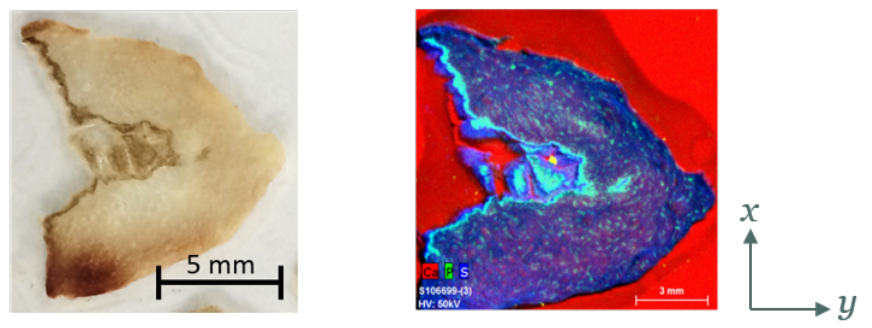

Figure 1. Photograph (left) and X-ray fluorescence (XRF) image (right) of the glass-backed $\sim 100$ um heterotopic ossification (HO) bone sample under investigation.

\section{ALGORITHMIC METHODS}

\subsection{Kramers-Kronig Algorithm}

Terahertz time domain spectroscopy (TDS) in reflection configuration involves the measurement of both the amplitude and phase of a terahertz pulse reflected from a sample and reference (usually a perfect electric conductor (PEC), but glass in this work), see Fig. 2. The extraction of biometric information fundamentally relies on the accurate retrieval of these electric field properties. The high sensitivity of the phase means that exact placement of the sample and reference planes is crucial, a task which is experimentally non-trivial at terahertz frequencies. Any displacement between the planes results in an additional phase in the measurements, which in turn, distorts the extracted dielectric properties. Considerable effort has gone into experimentally overcoming these challenges. ${ }^{13}$ Limitations of these methods has meant researchers have turned to post-processing approaches, specifically, a Kramers-Kronig approach. ${ }^{14,15}$
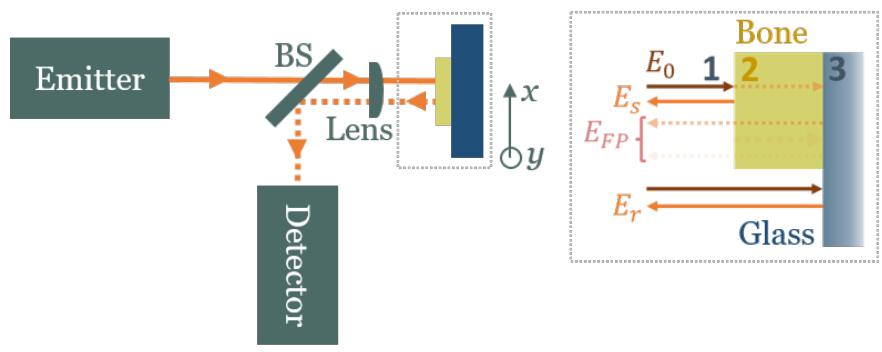

Figure 2. Experimental set-up of the THz-TDS in reflection geometry. The terahertz pulse generated by the photoconductive antenna (PCA) emitter passes through the beam splitter (BS), focused by a TXP35 lens with focal length 35 $\mathrm{mm}$ and is incident on the bone sample. The reflected pulse is then collimated by the same TPX35 lens and reflected by the beam splitter to be detected by the PCA detector. The imaging is performed by scanning the bone sample in the $x y$-plane using a translation stage. The inset illustrates the path of the electric field, with $E_{0}, E_{s}, E_{r}$ and $E_{F P}$ denoting the incident, sample (bone), reference (glass) and Fabry-Pérot field terms.

The Kramers-Kronig relations are mathematical bidirectional equations that can be used to describe the fundamental, causal relation between the real and imaginary parts of the complex refractive index of a material, where the real and imaginary parts describe the dispersion and absorption properties. By implementing an iterative algorithm developed by V. Lucarini and colleagues ${ }^{15}$ which checks the self-consistency between the amplitude (real part) and phase (imaginary part) of the retrieved terahertz electric field, one can obtain the phase offset, and hence the displacement between the sample and reference planes.

The HO bone samples under investigation sit on the surface of a glass slide, as illustrated in Fig 2. Through retrieval of the displacement between the sample and reference planes, one can determine the thickness of the sample through application of the algorithm. Fig. 3 presents the thickness of the bone slice shown in Fig. 1 obtained through mapping the topography of the bone by lasing profiling and through calculation of the phase offset through application of the Kramers-Kronig relations. One can observe good consistency between the two 
in the bulk region of the sample, confirming the reliability of the algorithm. The disagreement at the bone edges arises from the different scattering effects of the optical and terahertz probing beams. The successful determination of the sample thickness crucially enables modelling the Fabry-Pérot reflections within the sample towards removing such effects.

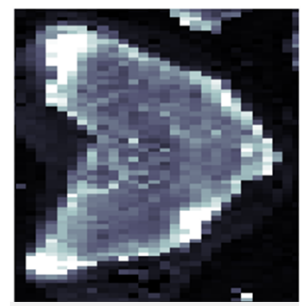

Topography

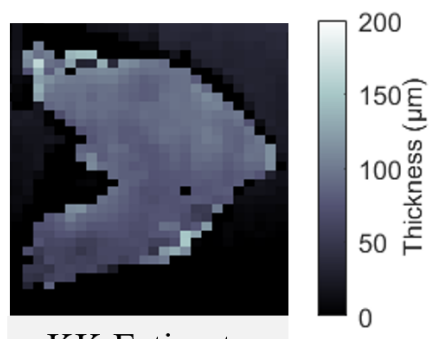

KK Estimate

Figure 3. Images of the thickness of the bone slice shown in Fig. 1 obtained through mapping the topography of the bone by lasing profiling and through calculation of the phase offset through application of the Kramers-Kronig relations.

\subsection{Fabry-Pérot Algorithm}

The temporal nature of TDS grants invaluable access to the temporal behaviour of materials and metamaterials. ${ }^{16}$ For optically thin samples, however, it comes with its limitations. For a sample of given thickness, one would expect to observe the terahertz pulse reflected by the first interface, followed by reflections of the pulse within the sample, termed Fabry-Pérot reflections. As the sample thickness is reduced, the reflected pulses are detected closer together in time, until they overlap. Resolving reflected pulses has been a significant challenge for researchers, resulting in the development of post-processing techniques in both the temporal and frequency domains. This contribution presents the development of a computationally simplistic algorithm designed to overcome these limitations through the mathematical modelling of the Fabry-Pérot reflections in the frequency domain. The reduced computational complexity and versatility over existing algorithms ${ }^{10}$ enables more accessible implementation for clinical use.

As illustrated in the inset of Fig. 2, the field, assumed to be a normally incident linearly polarised plane wave, is modelled for a thin sample of thickness $L$ (layer 2) fixed to a reference sample (layer 3 ). From the Fresnel coefficients, the transfer function, defined as the ratio of the sample and reference fields is given by

$$
H(\omega)=\frac{1}{P_{1}^{2} R_{13}}[R_{12}+\underbrace{\left\{T_{12} P_{2}^{2} R_{23} T_{21}+T_{12} P_{2}^{4} R_{23}^{2} R_{21} T_{21}+\ldots\right\}}_{\text {Fabry-Pérot terms }}],
$$

where $\omega$ is the angular frequency, $T$ and $R$ are the reflection and transmission Fresnel coefficients, respectively, and $P$ is the propagation term, given by $T_{12}(\omega)=\frac{2 \tilde{n}_{1}}{\tilde{n}_{1}+\tilde{n}_{2}}, R_{12}(\omega)=\frac{\tilde{n}_{1}-\tilde{n}_{2}}{\tilde{n}_{1}+\tilde{n}_{2}}$ and $P_{1}(\omega, L)=\exp \left(-i \tilde{n}_{1} \omega L / c\right)$, where 1 and 2 subscripts indicate the material layer.

Providing the dielectric response of the reference material is known, the properties of the sample can be retrieved through comparative approach. The modelled transfer function is calculated for a range of estimated refractive indices $n_{2}$ and extinction coefficients $\kappa_{2}$, where $\tilde{n}=n+\kappa i$. The output dielectric properties are given by minimising the L2 norm between the amplitude and phase of the measured and modelled transfer functions.

\section{RESULTS AND DISCUSSION}

Fig. 4 presents the application of the hybrid algorithm to a second $\sim 100 \mu \mathrm{m}$ HO bone slice, photographed in the bottom left. The top left image presents the peak-to-peak value of the electric field, while the top right and bottom right panels present multi-frequency images of the extracted refractive indices and extinction coefficients, post-application of the algorithm. 

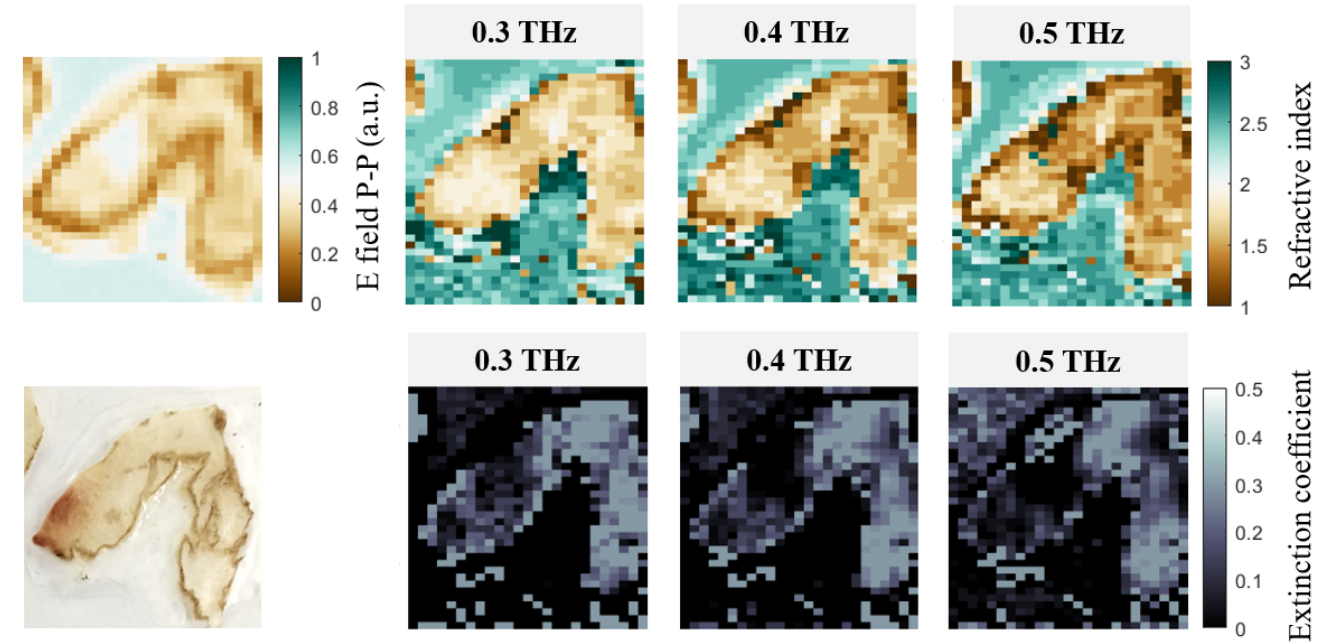

\section{$0.3 \mathrm{THz}$}

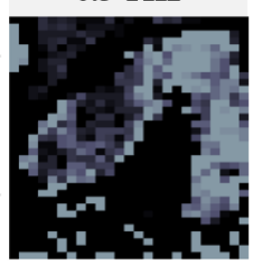

$0.4 \mathrm{THz}$

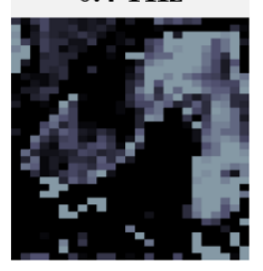

$0.5 \mathrm{THz}$

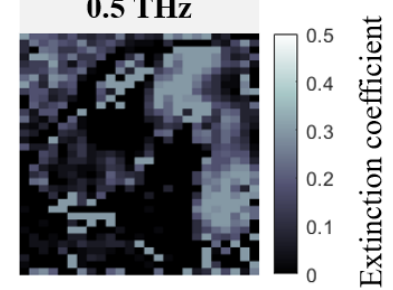

Figure 4. Terahertz images of a glass-backed $\sim 100 \mu \mathrm{m}$ HO bone slice, photographed in the bottom left. The top left image presents the peak-to-peak value of the electric field, while the top right and bottom right panels present images of the extracted refractive indices and extinction coefficients at $0.3 \mathrm{THz}, 0.4 \mathrm{THz}$ and $0.5 \mathrm{THz}$, post-application of the algorithm.

From the XRF images (see Fig. 1), the bulk of the samples were identified as collagen. TDS measurements of commercially available collagen provided dielectric properties consistent with the images presented in Fig. 4, with small differences attributed to differences in water content due to sample preparation.

These results demonstrate the success of the hybrid algorithm for application to thin, biological samples. This shows great promise for application to complex multilayered samples, for prospective use in a clinical environment.

\section{CONCLUSION}

Experimental challenges limiting the development of biometric terahertz imaging have been addressed through application of a hybrid algorithm. Combination of the application of the Kramers-Kronig relations and modelling of Fabry-Pérot reflections has enabled the successful extraction of dielectric properties from HO bone samples. This shows great promise for biometric imaging of samples within a clinical environment, while the versatility of the approach opens up the possibility for application to biological samples of increased complexity.

\section{APPENDIX A. EXPERIMENTAL METHOD}

Imaging was performed using an all fiber-coupled terahertz time-domain spectrometer TERA K15 from Menlo Systems with lock-in detection. ${ }^{17}$ The time-constant was $300 \mu$ s and the temporal length of the waveforms was $52 \mathrm{ps}$, providing a spectral resolution of $15 \mathrm{GHz}$. The system was set-up in standard reflection configuration with a Si beam-splitter, as shown in Fig. 2. The field was normally incident and focused on the sample using a TPX35 lens with $35 \mathrm{~mm}$ effective focal length. Single pixel scanning was used, through translation of the sample fixed to an $x y$-translation stage, with steps of $0.5 \mathrm{~mm}$.

\section{ACKNOWLEDGMENTS}

The authors would like to thank V. Lucarini et al. for the use of their Kramers-Kronig MATLAB scripts. ${ }^{18}$ This work was supported in part by the EPSRC [Grant Nos. EP/S018395/1 and EP/V001655/1] and the Royal Society [Grant Nos. IES/R3/183131 and IEC/NSFC/191104]. S. Freer was supported by the University of Birmingham [PhD studentship UKRI Project Reference 2137478]. S. M. Hanham and M. Navarro-Cía were supported by the University of Birmingham [Birmingham Fellowship]. 


\section{REFERENCES}

[1] Carpintero, G., Garcia-Munoz, E., Hartnagel, H., Preu, S., and Raisanen, A., [Semiconductor Terahertz Technology: Devices and Systems at Room Temperature Operation], Wiley (2015).

[2] Guerboukha, H., Nallappan, K., and Skorobogatiy, M., "Toward real-time terahertz imaging," Adv. Opt. Photon. 10, 843-938 (2018).

[3] Son, J.-H., [Terahertz Biomedical Science and Technology], CRC Press (2014).

[4] Jepsen, P., Cooke, D. G., and Koch, M., "Terahertz spectroscopy and imaging - modern techniques and applications," Laser Photonics Rev. 5, 124 (2011).

[5] Stringer, M. R., Lund, D. N., Foulds, A. P., Uddin, A., Berry, E., Miles, R. E., and Davies, A. G., "The analysis of human cortical bone by terahertz time-domain spectroscopy," Phys. Med. Biol. 50, 3211 (2005).

[6] Bessou, M., Chassagne, B., Caumes, J. P., Pradère, C., Maire, P., Tondusson, M., and Abraham, E., "Threedimensional terahertz computed tomography of human bones," Appl. Opt. 51(28), 6738-6744 (2012).

[7] Lindley-Hatcher, H., Hernandez-Serrano, A. I., Sun, Q., Wang, J., Cebrian, J., Blasco, L., and PickwellMacPherson, E., "A robust protocol for in vivo thz skin measurements," J. Infrared Milli. THz Waves 40, 980-989 (2019).

[8] Lucarini, V., Ino, Y., Peiponen, K. E., and Kuwata-Gonokami, M., "Detection and correction of the misplacement error in terahertz spectroscopy by application of singly subtractive kramers-kronig relations," Phys. Rev. B Condens. Matter Mater. Phys. 72, 125107 (2005).

[9] Vartiainen, E. M., Ino, Y., Shimano, R., Kuwata-Gonokami, M., Svirko, Y. P., and Peiponen, K.-E., "Numerical phase correction method for terahertz time-domain reflection spectroscopy," J. Appl. Phys. 96, 4171-4175 (2004).

[10] Duvillaret, L., Garet, F., and Coutaz, J. L., "A reliable method for extraction of material parameters in terahertz time-domain spectroscopy," IEEE J. Sel. Top. Quantum Electron. 2, 739-745 (1996).

[11] Naftaly, M., [Terahertz Metrology], Artech House (2015).

[12] Freer, S., Sui, C., Hanham, S. M., Grover, L. M., and Navarro-Cía, M., "A hybrid reflection retrieval method forterahertz dielectric imaging of human bone," Biomedical Opt. Express (Accepted 2021).

[13] Nashima, S., Morikawa, O., Takata, K., and Hangyo, M., "Measurement of optical properties of highly doped silicon by terahertz time domain reflection spectroscopy," Appl. Phys. Lett. 79, 3923 (2001).

[14] Peiponen, K. E., Lucarini, V., Saarinen, J. J., and Vartiainen, E., "Kramers-kronig relations and sum rules in nonlinear optical spectroscopy," Appl. Spectrosc. 58, 499-509 (2004).

[15] Lucarini, V., Bassani, F., Peiponen, K. E., Saarinen, J. J., Socie, C., and Fisica, I. D., "Dispersion theory and sum rules in linear and nonlinear optics," La Rivista del Nuovo Cimento 26, 1 (2003).

[16] Freer, S., Camacho, M., Kuznetsov, S. A., Boix, R. R., Beruete, M., and Navarro-Cía, M., "Revealing the underlying mechanisms behind te extraordinary thz transmission," Photon. Res. 4, 430-439 (2020).

[17] Freer, S., Gorodetsky, A., and Navarro-Cía, M., "Beam profiling of a commercial lens-assisted terahertz time domain spectrometer," IEEE Trans. Terahertz Sci. Technol 11(1), 90-100 (2021).

[18] Lucarini, V., Saarinen, J. J., Peiponen, K.-E., and Vartiainen, E. M., [Kramers-Kronig Relations in Optical Materials Research], Springer-Verlag Berlin Heidelberg (2005). 